\title{
FPGA Implementation of Oxygen Level Monitoring in Oxygen Cylinder
}

\author{
Santhosh $\mathrm{KV}^{1}$ and B K Roy ${ }^{2}$ \\ ${ }^{1}$ Dept of ICE, MIT Manipal, ${ }^{2}$ Dept of EE, NIT Silchar \\ ${ }^{1}$ kv.santhu@gmail.com, ${ }^{2}$ bkr_nits@yahoo.co.in
}

\begin{abstract}
This paper aims at designing an oxygen level monitoring technique in an oxygen cylinder. The amount of oxygen present inside an oxygen cylinder is a very vital information when such cylinder is in use as life saving measure to a critical patient. In this paper, it is proposed to measure oxygen level using pressure and temperature sensors. Conditioned output of these sensors is connected as input to ARM microcontroller. ARM is programmed to display the actual pressure of oxygen cylinder in terms of numerical values and also in terms of fuzzy variables. A buzzer is also used as indicator to caution the attendants of patients whenever the level of oxygen is below a pre-decided value. The signal from the cylinder is further transmitted to the monitoring station through wireless communication module. Graphical display is used at monitoring station to indicate pressure of all oxygen cylinders to initiate actions like use cylinders which are in good conditions, replacement of empty cylinders with filled ones, etc. Experimental results show that, the aims set for this work are achieved satisfactorily.
\end{abstract}

Keywords: Oxygen Cylinder, Pressure sensor, Temperature Sensor, LabVIEW, Fuzzy logic, Fault detection, ARM micro-controller

\section{Introduction}

Oxygen is our primary life-support. The air we breathe is so vital that without it we would rapidly die. Clean air is made up of several gases of which Oxygen is the most important to us. Clean air contains 19\%-21\% Oxygen. The generation and maintenance of all our body processes are supported by four basic life-support components: carbohydrates, water, proteins, and energy. Most Scientists agree that oxygen is actually the over-riding key ingredient in all four life-support components. The oxygen concentration in a healthy human body is approximately three times than that of air. When body oxygen falls to extremely low levels for prolonged periods of time, the body may become a breeding ground for harmful bacteria, viruses, fungi, parasites, and other infectious agents. Most of these are anaerobic, i.e., they cannot live in an oxygen-rich environment. Some research indicates that when the oxygen content of the body is within the normal level, infectious microorganisms have a more difficult time for breeding and multiplying. The partial pressure of oxygen in normal blood should be approximately 97\%. Within each red blood cell there are iron-rich hemoglobin molecules. Approximately $97 \%$ of the oxygen carried to the cells is attached to these hemoglobin molecules and 3\% of the oxygen supply dissolved in the blood plasma. When the blood oxygen levels remain low for extended periods of time, the cells cannot get an adequate and consistent supply of oxygen and there may be difficulty in resisting the invasion of microorganisms resulting in lessening of natural life-support [1-4].

Under this circumstance, when lungs of a human body fail to take appropriate amount of oxygen inside the body, an oxygen cylinder is used to pump oxygen to the human body. Thus an appropriate arrangement for supply of oxygen from an oxygen cylinder can be termed as one of the live saving device $[5,6]$. It is very important to continuously 
monitor the status of such devices. Seldom, it is seen that the amount of oxygen content inside the cylinder is unnoticed. When the patient's feels uncomfortable due to nonavailability of oxygen in cylinder, suddenly there are rushes and may lead to severe consequences.

Several researchers have discussed about pressure measurement techniques like [7], a capacitance pressure sensor is used to measure in-cylinder pressure of an engine. Uses of rogowski coil for pressure measurement in a cylinder is discussed in [8]. In [9], dynamic pressure of hollow cylinder is measured by measuring the potential difference between external and internal piezo-electric sensor. Fiber optic sensor is used to measure the dynamic cylinder pressure in a spark ignition engine is reported in [10]. In [11], a Fiber Bragg grating sensor is used to measure the pressure in a cylinder. In [12], a pressure sensor is used for measurement of pressure in a cylinder. From the survey of available technique it is clear that many techniques are reported for measurement of pressure in a cylinder. But not much of research is carried on pressure/level measurement of oxygen cylinders. Though the oxygen cylinder is life saving device the techniques still adopted is analogus and crude. Thus an automated technique would benefit a lot for the community.

So, this paper proposes a technique which will keep track of the oxygen levels locally and centrally in the cylinders which are in use at any point of time in a hospital. It also does the local measurement of oxygen level in terms of numerical indicators and fuzzy variables, and a buzzer beeps when the oxygen level in the oxygen cylinder is dropped below a pre-decided value, so that attendant of a patient can be alert along with on duty nurses and ward-boys for immediate backup. The proposed paper is an extension of the earlier work [13], in which a method for measuring the level of oxygen of a cylinder was discussed. For this purpose multi sensor fusion architecture is used comprising of pressure and temperature sensors. The output of this sensor is interfaced to ARM microcontroller. ARM is programmed to calculate the actual level of oxygen in the cylinder in terms of pressure. Level output is also displayed in terms of fuzzy variables so that the common person will understand. A buzzer beeps when the pressure drops below a predefined lower level. Further, oxygen level is also transmitted to the monitoring station using wireless communication to keep track of all cylinders in use at any point of time. Since oxygen cylinder is portable, power needed for the proposed device is derived from a battery. It is important to monitor the condition of battery; this is also taken care in the proposed technique. At the monitoring station, LabVIEW software is used to accept data and graphically display of level in oxygen cylinder are shown on the front panel.

The paper is organised as follows: after introduction in Section-1, Section-2 discusses the need and objective of the proposed works. Section-3 discusses details of the experimental model used for proposed work, followed by proposed solution in Section-4, and results and analysis is reported in Section-5. Finally, conclusion is given in Section-6.

\section{Objective}

From survey of available techniques it is found that many reported work have been discussed for measurement of pressure. But no attempts have been made to design an automated technique for level measurement in oxygen cylinder. In this regard this paper proposes a technique to design a technique to measure the oxygen level in cylinder using pressure and temperature sensor having the following characteristics:

- Process signal from sensors and display the actual pressure of oxygen cylinder.

- Display level of oxygen in terms of pressure using fuzzy variables, like 'GOOD', 'CHECK' and 'REPLACE'.

- Activate a buzzer once oxygen level in the oxygen cylinder drops below predefined level.

- Communicate with monitoring station using wireless communication. 
- Display status of all oxygen cylinders at the monitoring station in use at any point of time in the hospital.

\section{Experimental Setup} 2.

To achieve the objectives a system is designed as in block diagram Figure 1 and Figure

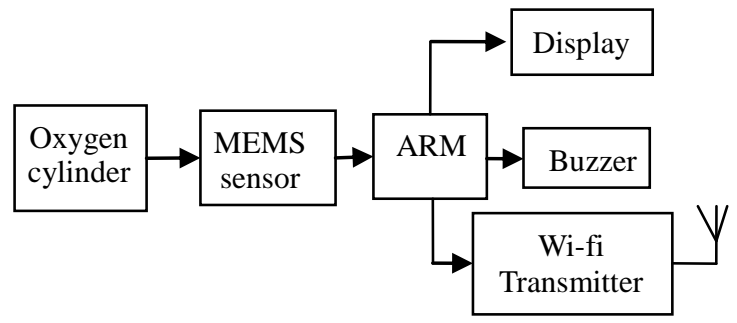

Figure 1. Block Diagram of the Transmitter Block

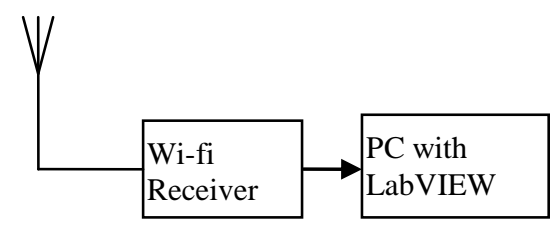

Figure 2. Block Diagram of the Receiver Block at Monitoring Station

\subsection{MEMS Pressure Sensor}

SX series of pressure sensors provides a cost effective method of measuring pressures. These sensors are specifically designed to be used with non-corrosive and non-ionic media, such as air and dry gases $[14,15]$.

\subsection{Temperature Sensor}

AD8496 is precision instrumentation amplifiers with thermocouple cold junction compensators on an integrated circuit. They produce a high level $\left(5 \mathrm{mV} /{ }^{\circ} \mathrm{C}\right)$ output directly from a thermocouple signal by combining an ice point reference with a precalibrated amplifier. They can be used as standalone thermometers $[15,16]$.

\subsection{Signal Conditioning Circuit}

The output voltages from both the sensors are of the order of few milli volts. An amplifier is designed to amplify and condition the signal to a range of $0-5 \mathrm{~V}$.

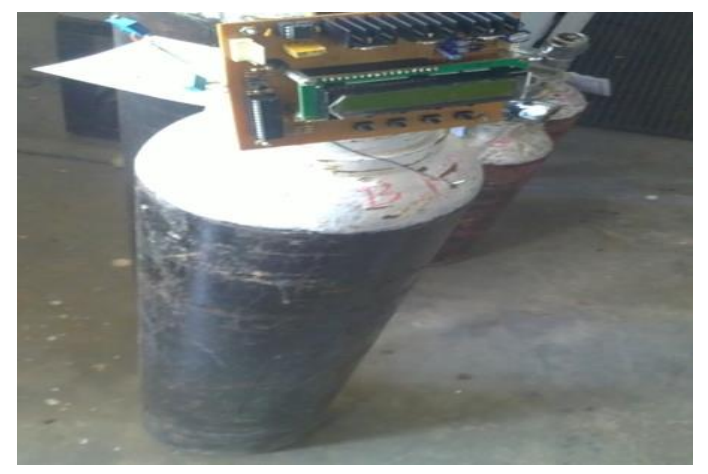

Figure 3. Experimental Setup of the Proposed Technique 


\section{Solution}

Implementation model of proposed measurement technique is carried over at two distinct places first one is locally placed at the cylinder and other at remote location. Proposed implementation on the cylinder is shown in Figure 3. Pressure \& temperature sensors are placed at nozzle of oxygen cylinder to measure pressure head of cylinder. Once, pressure and temperature are sensed, it produces voltages output which varies with oxygen pressure. Signals thus obtained are amplified and fed as inputs to an ARM 7 controller through two analog input pins. ARM controller is programmed to acquire input signals from these sensors. Once signals are acquired it is processed to display the actual pressure using a LCD display. Processed value is fuzzified using fuzzy logic algorithm to display output pressure of oxygen cylinder. A buzzer is connected which activates as soon as pressure drops below a preset pressure level. The wi-fi transmitter module is designed and connected to RX/TX pin of ARM micro-controller to transmit the oxygen level data to the monitoring station. Since the entire setup will be installed on cylinder, battery is used to power the circuit. A circuit is also designed to indicate the output voltage of battery.

It is clear from Figure 1 that the signal corresponding to the oxygen level is sensed by sensors. The outputs of these sensors are fed to the ARM 7 processor chip for processing. The micro-controller on receiving the signals carries out the following tasks.

* Acquire the signals from temperature and pressure sensor.

* Calculate the pressure corresponding based on the Transducer Electronic Datasheet (TEDs) of temperature and pressure responses and display the same on LCD display.

* Implementation of fuzzy logic algorithm to display the level of oxygen cylinder in terms of fuzzy variables like 'GOOD', 'CHECK' and 'REPLACE'.

The fuzzy tool box is used to design and display the level of oxygen in cylinder in terms of three fuzzy terms. These are 'GOOD' - meaning the oxygen pressure is in desired range of utilization, 'CHECK' - meaning oxygen pressure is low and need to be checked, and 'REPLACE' - meaning oxygen pressure is below designated range of utilization and need to be replaced immediately. A fuzzy logic tool box is used to implement fuzzy logic algorithm [17-19]. Figure 4, 5, and 6 shows the input and output membership functions used for implementation of fuzzy logic. Table 1 shows the fuzzification table used for the same. Figure 7 shows the surface graph of the implemented fuzzy block.

* Activate the buzzer when the pressure drops below the pre decided value.

* Send the data through UART port which is connected with wi-fi module for transmitting to remote station.

* At remote location received data is processed using LabVIEW software and HMI SCADA system is designed for monitoring and control.

Table 1. Fuzzy Table

\begin{tabular}{|c|c|c|c|}
\hline \multirow{2}{*}{$\begin{array}{c}\text { pressure } \\
\text { temp }\end{array}$} & \multicolumn{3}{|c|}{ o/p } \\
\cline { 2 - 4 } & HT & MT & LT \\
\hline VHP & good & good & Good \\
\hline HP & good & good & Good \\
\hline MP & good & check & Check \\
\hline LP & check & check & Check \\
\hline VLP & check & replace & replace \\
\hline
\end{tabular}




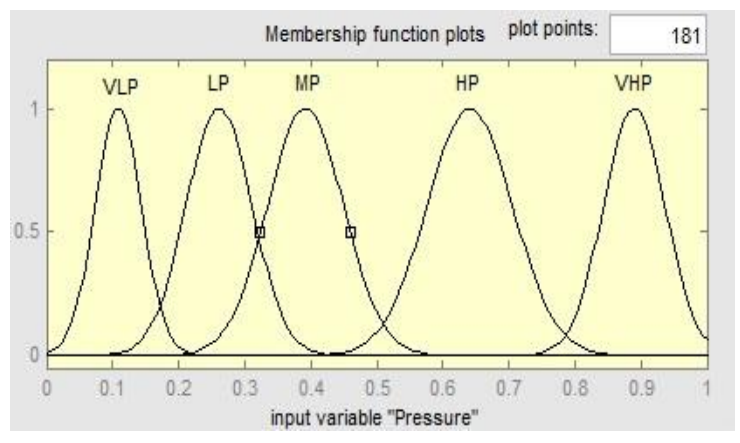

Figure 4. Input Membership Function for Pressure Input

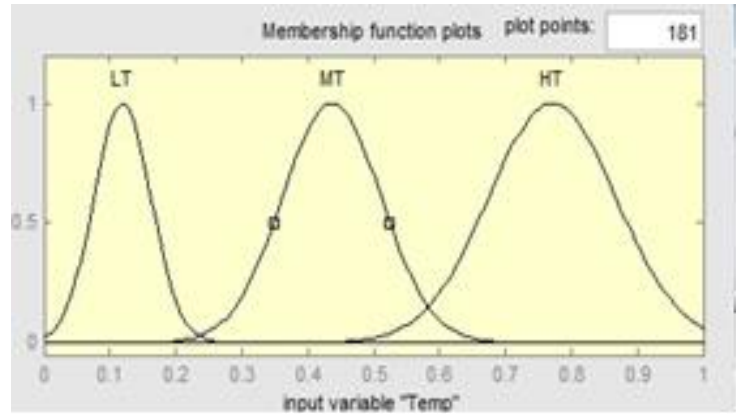

Figure 5. Input Membership Function for Temperature Input

The monitoring station/ remote center can be at nurses' duty room/ doctors' duty room. The status of oxygen pressure in all the cylinders in use are displayed at monitoring station for effective and timely follow up actions. At remote station an operator can check the status of all the cylinders present in hospital area. Remote center uses NI Wi-Fi DAQ card to accept all data from different cylinders. Once the data is received it is acquired to the PC, LabVIEW platform is used to program desired objective. LabVIEW platform consists of two panels, namely the front panel and block diagram panel. The panel where the user can control or get the indications is known as front panel and the where the actual program is coded is called block diagram panel. LabVIEW programs is not textual, these are programmed by the interconnections of palates (defined functional blocks) [20, 21]. Figure 8 shows front panel VI of proposed work, it consists of a 'start' button to control the initiation of the process, a cluster of indicator/control switch which indicate the status of each cylinder.

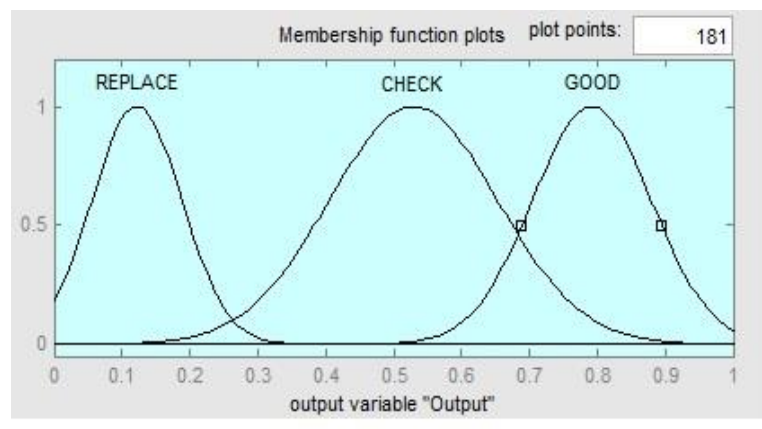

Figure 6. Output Membership Function 


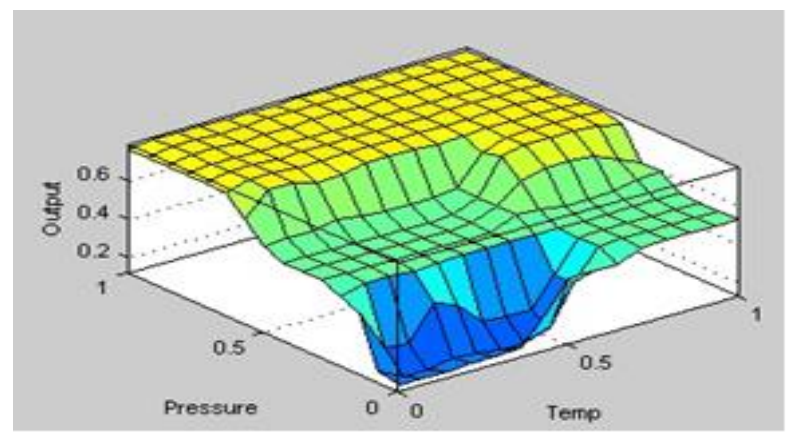

Figure 7. Surface Graph

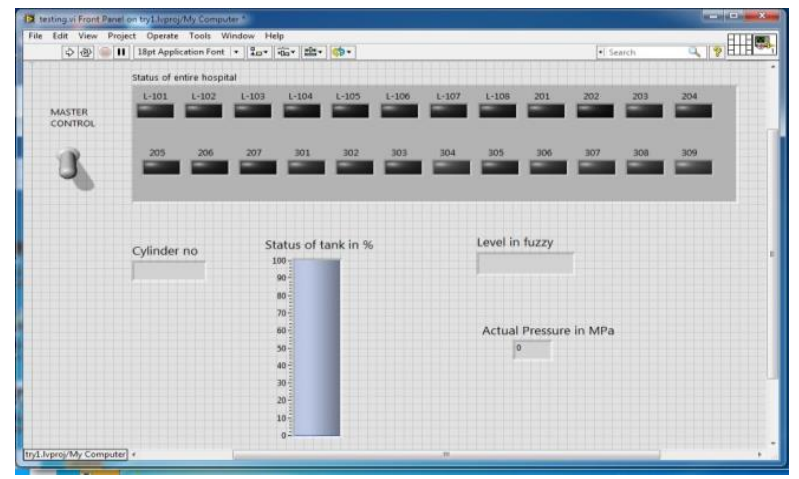

Figure 8. Front Panel of Monitoring Station

Array of indicators/switch represent status of all cylinders in the hospital, each indicator is given a number which represents a cylinder in the particular room. These indicators display the status of cylinders in terms of colors i.e., 'Green' if OK, 'Yellow' if action needed, 'Red' for cylinder which needs to be replaced, and 'Black' if in case cylinder is not in use. On selecting a specific indicator, complete detail of cylinder in terms of actual pressure, percentage of oxygen present, and output fuzzy variable will be displayed.

\section{Results and Analysis}

Once the whole process of oxygen level monitoring system is designed it is subjected to test in real life. The process was tested to analyze performance of on board and remote station. For experimentation six cylinders were used and placed at different locations. Table 2 shows the results obtained from proposed block and existing standard measuring device. Display at the monitoring station is shown in Figure 9, 10, and 11.

It is seen from Figure 9, 10, and 11, that level of oxygen cylinder is monitored using numerical display and visual display. The unit of the numerical display can be set as per requirement of hospital staff. Visual display is very useful, since it gives a feeling on the status of level of oxygen available in the cylinder. Duty staff needs more attention when the time for replacement is knocking at the door. A warning message as 'CHECK' along with a glow of LED indicates this critical time.

The results in Table-2 show that proposed technique produces result which matching with those is obtained using a standard pressure measurement instrument. Thus, it shows that the proposed monitoring technique works satisfactorily. Buzzer was 'ON' when the pressure level was less than the predefined value of 3.5MPa. 


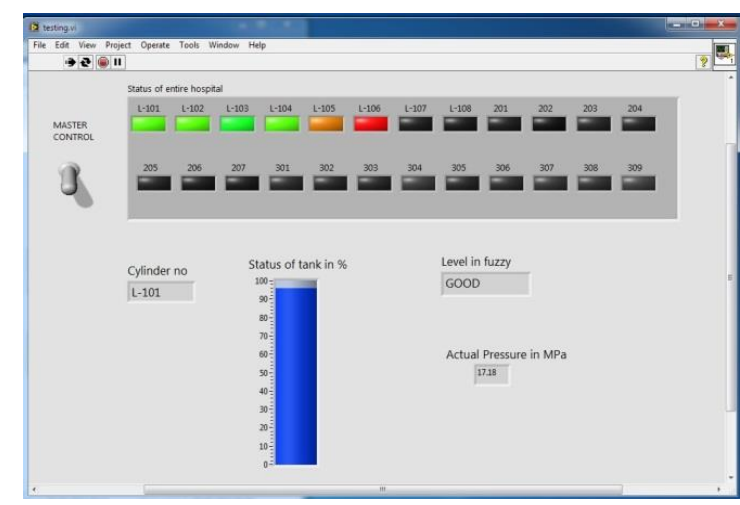

Figure 9. Result at Monitoring Station

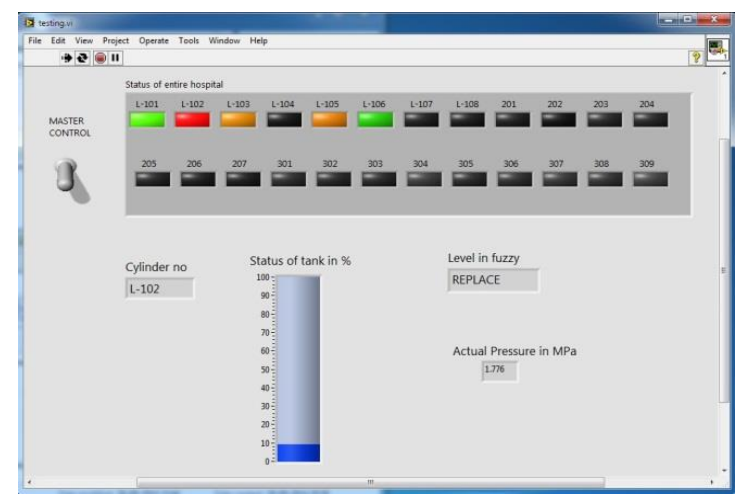

Figure 10. Result at Monitoring Station

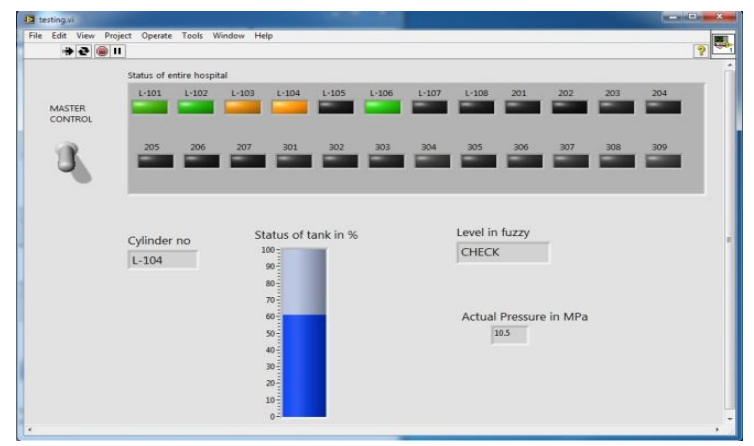

Figure 11. Result at Monitoring Station

Table 2. Result of the Proposed Measuring Technique

\begin{tabular}{|c|c|c|c|c|}
\hline $\begin{array}{c}\text { Sl. } \\
\mathrm{N} \\
\mathrm{o}\end{array}$ & $\begin{array}{c}\text { Existing } \\
\text { instrum } \\
\text { ent } \\
(\mathrm{MPa})\end{array}$ & $\begin{array}{c}\text { Proposed } \\
\text { technique } \\
(\mathrm{MPa})\end{array}$ & Buzzer & Fuzzy o/p \\
\hline 1 & 17.01 & 17.02 & Off & GOOD \\
\hline 2 & 10.35 & 10.34 & Off & GOOD \\
\hline 3 & 8.78 & 8.78 & Off & CHECK \\
\hline 4 & 5.33 & 5.31 & Off & CHECK \\
\hline 5 & 3.25 & 3.28 & On & REPLACE \\
\hline 6 & 1.68 & 1.64 & On & REPLACE \\
\hline
\end{tabular}




\section{Conclusions}

Implementation of oxygen pressure monitoring in an oxygen cylinder is presented in this paper. The comparative result of level measurement by the proposed technique shows that the measurement is done accurately. Various indicators are used locally and centrally.

The user friendly display and alert at patient attendant's end and at monitoring station is expected to be highly beneficial to reduce the causalities due to non-replacement of oxygen cylinder at appropriate time. Implementation of such a system at big hospitals and hospital where the ratio between patient and nurses/doctors is high will be beneficial with respect to effective utilization of man power and life saving device.

Real life implementation at some hospital will be within our scope in future.

\section{References}

[1] L. Bertoletti, P. Mismetti and H. Decousus, "Trends in Cause-Specific Mortality in Oxygen-dependent", American Journal of Respiratory Critical Care Medicine, vol. 181, no. 8, (2010), pp. 1032-1036.

[2] H. M. Womble, R. P. Johnston, R. M. Schwartzstein and D. H. Roberts, "The Effect of Supplemental Oxygen on Maximal Consumption of Oxygen and Ventilatory Parameters in Chronic Obstructive Pulmonary Disease", American Journal of Respiratory Critical Care Medicine, vol. 181, no. 1, (2010), pp. A3572-A3577.

[3] H. M. Womble, R. M. Schwartzstein, R. P. Johnston and D. H. Roberts, "Effect of Therapeutic Hyperoxia on Maximal Oxygen Consumption and Perioperative risk Stratification in Chronic Pulmonary Disease”, Lung Jr., vol. 190, no. 3, (2012), pp. 263-269.

[4] B. R. Celli and W. Macnee, "Standards for the Diagnosis and Treatment of Patients with COPD: A Summary of the ATS/ERS position paper", European Respiratory Journal, vol. 23, (2004), pp. 932-946.

[5] B. O. Neill, J. M. Mahon and J. Bradley, "Short-burst Oxygen Therapy in Chronic Obstructive Pulmonary Disease”, Respiratory Medicine, vol. 100, no. 7, (2006), pp. 1129-1138.

[6] J. K. Stoller, R. J. Panos, S. Krachman, D. E. Doherty and B Make, "Oxygen therapy for patients with COPD: current evidence and the long-term oxygen treatment trial", Jr. Chest, vol. 138, no. 1, (2010), pp. 179-187.

[7] L. Chen and M. Mehregany, "A Silicon Carbide Capacitve Pressure Sensor for in-cylinder Pressure Measurement", Sensors and Actuators A, vol. 145, (2008), pp. 2-8.

[8] X. Xuedong, C. Xude, X. Bing, Z. Yuan and Y. Guangzheng, "Design of Auto Cylinder Pressure Measurement System based on PCB Planar Rogowski Coil", Proceedings of International Conference on Measurement, Information and Control, Harbin, China, (2013) August.

[9] H. M. Wang, H. J. Ding, "Determination of dynamic Pressure on Piezoelectric Hollow Cylinder from Electrical Potential Difference Measurement", International Journal of Pressure Vessels and Piping, vol. 86, (2009), pp. 351-356.

[10] M. Wndekar and T. Kaminski, "Development of a Fiber-Optic Sensor for the Measurement of Dynamic Cylinder Pressure in Spark Ignition Engine", Proceedings of IEEE Sensors Conference, CA, USA, (2005) October.

[11] B.-J. Peng, Y. Zhao, J. Yang and M. Zhao, "Pressure sensor based on a Free Elastic Cylinder and birefringence effect on an FBG with temperature-compensation", Measurement, vol. 38, (2005), pp. 176-180.

[12] S. Fei and K. Deren, "Quasi-static Absolute Calibration Method of Piezoelectric Sensors for Pressure Measurements", Proceedings of International Conference on Electric Information and Control Engineering, Wuhan, China, (2011) March.

[13] K. V. Santhosh, B. K. Roy and P. Kumar Bhowmik, "Oxygen Level Monitoring in an Oxygen Cylinder", Proceedings of International Conference on Engineering, Science and Managament, Nagappttinam, India, (2012) April.

[14] S. Beeby, "MEMS Mechanical Sensor", Artech House, (2002).

[15] T.-R. Hsu, "MEMS \& Microsystems Design \& Manufacture and Nanoscale Engineering", Wiley, (2002).

[16] K. Iniewski, "Smart Sensor for Industrial Applications”, CRC Press, (2013).

[17] E. Alpaydın, "Introduction to machine learning", (2nd ed.). Cambridge- MIT Press, (2010).

[18] S. Rajasekaran and G. A. Vijayalaksmi, "Neural Network, Fuzzy Logic, and Genetic Algorithms Synthesis and Applications", Prentice Hall India, (2005).

[19] T. Ross, "Fuzzy Logic with Engineering Applications", John Wiley \& Sons Inc., (2004).

[20] L. Mahesh, Chugani, LabVlEW Signal Processing, Prentice-Hall India, (1998).

[21] R. Bishop, Learning with LabVIEW 7 Express", Prentice Hall, New Jersey, (2004). 


\section{Authors}

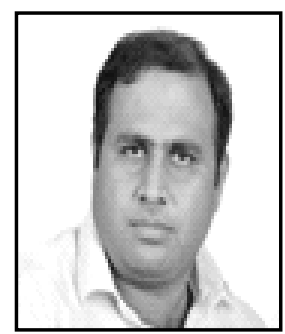

Santhosh KV is presently working as a faculty in Instrumentation and Control Engineering department of Manipal Institute of Technology, Manipal, India. Santhosh received his Bachelors and Masters from Visvesvaraya Technological University, Belgaum in year 2004 and 2006 respectively. He obtained $\mathrm{PhD}$ in the year 2013 from National Institute of Technology Silchar. His research interests include intelligent measurement, automation, sensor fusion, and image processing. Email: kv.santhu@gmail.com

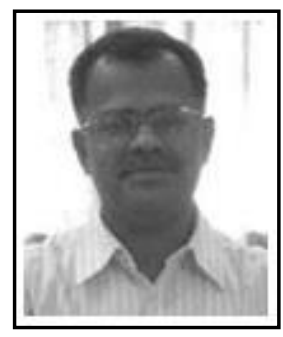

B. K. Roy received the B. E. degree in Electrical Engineering from National Institute of Technology (formerly known as Regional Engineering College) Silchar, Assam, India, in 1985. M. Tech in Control System and Ph.D. degree in 'Fault Detection and Diagnosis of Dynamical Systems' were received from IIT Kharagpur, India in 1989 and 1998 respectively. Currently, he is a Professor with the National Institute of Technology, Silchar, Assam, India. His research interest includes the Control Systems, Nonlinear dynamics and Chaos, Virtual Instrumentation, Fault Detection and Diagnosis of Dynamical Systems, Image Processing and Computer Vision, and Industrial Automation. Email: bkr_nits@yahoo.co.in 
International Journal of Bio-Science and Bio-Technology

Vol.7, No.5 (2015) 